\title{
REFLECTIONS
}

\section{Reflections on six years in paediatric ART}

H A Moore

Corresponding author: H A Moore (hazel.ann.moore@gmail.com)

Dr Hazel Ann Moore is a general practitioner based in Khayelitsha, Cape Town, South Africa

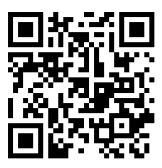

As part of my work at a primary care clinic in Khayelitsha, I started the paediatric arm of the antiretroviral therapy (ART) service in our clinic six years ago. When I first started, many children were being cared for by family members or foster carers because their mothers had died. This is now the exception, as most mothers receiving ART survive. Today, the numbers of patients are still relatively small compared with those in many other programmes, although there are now over 210 children, with a further $10-15$ adolescents having graduated to the adult ART section. Over time, this had the advantage of allowing me to get to know the families and essentially to function as their general practitioner. In terms of the model of care, a doctor (myself) starts all the small children on ART and then passes their care on to the nursing sisters when they are stable and about 3 years of age; I continue to see the children with clinical problems until they are stabilised. This approach is obviously not possible in many parts of our country, but has had many advantages.

Some of my reflections on spending time in primary care services for paediatric HIV include:

\section{Adherence and support}

I have cared for a lot of children from a local children's home who have made a brilliant control group, as they are all suppressed as a result of getting their medication on time every day. This demonstrates the importance of adherence to treatment regimens. I have even used the home temporarily for children who were failing their regimens - once suppressed, they were returned to their families, who had in the meanwhile been educated to ensure better adherence. One child, placed there voluntarily by her mother because of concerns about carer adherence, continued to show a detectable viral load. Having proven that it was not due to a lack of adherence, we could confidently place her on treatment with second-line drugs; she subsequently suppressed, indicating that she had developed resistance.

\section{Disclosure}

Disclosure is often a problem, by the mother to her family as well as disclosure to the child. I believe that we need ageappropriate support groups to assist in this. Small children grow up into big children and adolescents, and they need progressive disclosure so that they are fully informed about their status well before puberty. Disclosure is often a particular problem with the very young mother (who may be scared to tell her own mother her status), or the working mother (who may not want her employer to know and does not want to take time off work to come to the clinic; the family may be dependent on her work income and so she does not want them to worry about her health status).

\section{PMTCT}

In contrast to previous years, the prevention of mother-tochild transmission (PMTCT) of HIV programme is now so effective that few babies become HIV-positive; but those who do frequently pose real problems. They are often born to young mothers who have not disclosed their status and/or have poor home circumstances. These mothers need intense counselling and support, especially for the first few months, as there is a high drop-out rate, especially if they have not yet disclosed their status to their family. Mothers often have difficulty accepting the positive status of their child, as they no longer expect it.

\section{Mobile populations}

We serve a very mobile population who constantly transfer in and out of the clinic, with or without informing the clinic. Often clients are called away unexpectedly (e.g. to a funeral in another province) and they do not think about, or do not have time to come to the clinic for medication before departure. The National Health Laboratory Service (NHLS) is sometimes a good resource when trying to ascertain a client's previous history, as we can track where blood samples have been taken! I have found this helpful when trying to find out more about an abandoned child. This, however, illustrates the need for a national database or a Medicalert-type card where clients could voluntarily access their medication anywhere. They would also then be recorded as compliant by collecting their medication.

\section{Defaulters}

The computer programme that we utilise (TIER.net) has a system for following up defaulters, but in practice this is not well implemented for various reasons. One reason is the delay in data capturing. It would be helpful if each clinician had a desktop computer, to allow data to be updated during the consultation. I have kept my own computer records on my laptop, as well as an appointment book for consultations, which has helped me to keep track of my patients. I have found that I received a good response from those mothers/carers whom I texted personally quite soon after they defaulted (one week 
later) and I think this was partly because they felt a sense of personal interest from 'their doctor'. Once they have defaulted for a long time, they are often too embarrassed to return, but a text message, even at this late stage, may give them a reason to return to the clinic without losing face. It certainly does not help to get angry with defaulters as there is usually a very valid reason for defaulting, such as problems in the family, and these need to be dealt with as part of the greater model of care.

\section{Seeing the same clinician}

Seeing the same clinician at each visit has many advantages. If possible, this is a much better system as it leads to good clinician/patient/family interaction and usually results in better adherence. It can be organised to an extent by making appointments on certain days for specific clinicians.

\section{Neurological side-effects}

The neurological side-effects of HIV are considerable. Children with major impairment are appropriately referred to special schools, but those with minor impairment (and this is a large proportion of children receiving ART) remain in mainstream education with little, if any, assistance. There seems to be very little in place to help the children with minor impairment and this will need to be addressed urgently by educational authorities.

\section{Clubs for children receiving ART}

This would be of considerable value for stable children. Maybe, one for pre-schoolers, one for primary school and one for high school/ teenagers. Occasionally, it would be helpful to have carers and children together, and sometimes to have them separate. In this way, issues such as progressive disclosure could be dealt with in an age-appropriate manner, together with other issues that affect the specific age groups. Teenagers are a particular challenge. They need lots of support and interaction to prevent them from defaulting, as well as lots of input about sexuality and their responsibilities in this regard. Teens at school find it difficult to miss school to come to the clinic. A possible solution would be to evaluate the stable ones clinically only four times a year (in the school holidays) with their carer collecting their medication in between.

\section{Treating the entire family}

I have found it very helpful to treat both mother and child in the same consultation, essentially offering a one-stop-shop. This way, the mother's time in the clinic is minimised and the clinician gets to know the family circumstances. This is an essential part of treating patients as their social circumstances have a considerable impact on how they perform on treatment. In one particular case, I treat both parents and their child.

It has been interesting and stimulating to be in the field of HIV medication, particularly with regard to treatment in children. The management has come a long way, but it is an evolving field and there is a long way to go yet.

S Afr J HIV Med 2014;15(1):22-23. DOI:10.7196/SAJHIVMED.1020 